\title{
Dermatologische Kosmetik in der Praxis und im Institut
}

\section{Dermatological Cosmetics Within the Clinic or Institute}

\section{Gwendolyn Gemke}

\section{Zusammenfassung}

Die rechtlichen Rahmenbedingungen für die dermatologische Kosmetik in der ärztlichen Praxis und im Kosmetikinstitut haben sich in den vergangenen Jahren erheblich geändert. Ein gewerbliches Engagement niedergelassener Ärzte wird im Grundsatz anerkannt, hinsichtlich Art und Weise der Integration in die ärztliche Praxis sind jedoch weiterhin die teilweise liberalisierten Vorgaben des ärztlichen Berufsrechtes sowie steuerliche Besonderheiten, insbesondere die grundsätzliche Umsatzsteuerpflicht kosmetisch indizierter Leistungen, zu beachten.

\section{Novellierung der Musterberufsordnung}

Im Zuge des Gesundheitsmodernisierungsgesetzes und der Novellierung der Musterberufsordnung für Ärzte wurde das ärztliche Berufsrecht wesentlichen Strukturänderungen unterzogen. Die Landesärztekammern ziehen nunmehr mit der Umsetzung der Novellierungen in den Landesberufsordnungen nach. Auch die Rahmenbedingungen für ein gewerbliches Engagement des Dermatologen neben seiner Praxis haben sich damit verändert. Vorbei sind die Zeiten, in denen jede gewerbliche Tätigkeit unter Verweis auf das standesrechtlich verankerte Gebot der Freiberuflichkeit in Bausch und Bogen verurteilt wurde. Eine - bislang erste - Entscheidung des Landgerichtes Darmstadt vom 23. 3. 2004 sieht sogar die der Integration eines gewerblichen Anbots in die ärztliche Praxis für zulässig an, so weit im Übrigen eine ausreichende organisatorische Trennung gewährleistet ist (LG Darmstadt, Az: 12 O 563/03; nicht rechtskräftig). Sie setzt sich damit in Gegensatz zu früheren Entscheidungen anderer Gerichte (z.B. VG Köln, Urteil v. 24. 11. 2000, Az: 35 K 2910/00).

\section{Abstract}

The legal terms and conditions regarding dermatological cosmetics within medical clinics and institutes have changed a great deal in recent years. The involvement of established doctors in the business aspects of cosmetics is principally allowed, dependent upon the degree of this business integration within the clinic. This liberalization of the business integration of dermatologists critically examines the required income and profit taxes on aesthetic cosmetic services not otherwise connected to by the rendering of other medical services.

Bei dem Aufbau eines dermatologischen Institutes und der Integration kosmetischer Leistungen und eines Produktverkaufes sind jedoch weiterhin die Vorgaben des ärztlichen Berufsrechtes zu beachten, die im Interesse eines umfassenden Patientenschutzes aufgestellt wurden. Auch die steuerrechtlichen Implikationen sind bei der Planung zu berücksichtigten, um unerwartete Steuerfallen zu vermeiden.

\section{Freiberuflichkeit und Gewerbe}

Auch nach der Novellierung des ärztlichen Berufsrechtes gilt für die eigentliche ärztliche Tätigkeit der Grundsatz des $\S 1$ Abs. 1 Berufsordnung, wonach der ärztliche Beruf kein Gewerbe ist. Der Arzt ist verpflichtet, seine Tätigkeit vorrangig an den Geboten der Menschlichkeit und ärztlichen Ethik, kurz am Wohle der Patienten auszurichten. Ein generelles Verbot gewerblicher Tätigkeit neben der dermatologischen Praxis kann daraus nach einhelliger Rechtsprechen nicht abgeleitet werden. 


\section{Leistungsort Praxis}

Nach $§ 3$ Abs. 2 der Berufsordnung ist es dem Arzt untersagt, im Zusammenhang mit der Ausübung seiner ärztlichen Tätigkeit Waren und andere Gegenstände abzugeben oder unter seiner Mitwirkung abgeben zu lassen sowie gewerbliche Dienstleistungen zu erbringen oder erbringen zu lassen, soweit nicht die Abgabe des Produkts oder die Dienstleistung wegen ihrer Besonderheiten notwendiger Bestandteil der ärztlichen Therapie ist. Eine Integration des gewerblichen Angebotes in die ärztliche Praxis ist demnach nur für solche gewerblichen Leistungen denkbar, die in engem Zusammenhang mit der ärztlichen Therapie stehen. Das Angebot gewerblicher Dienstleistungen wie Maniküre oder dekorative Kosmetik und damit verbundene Produkte ist dagegen in der Praxis unzulässig. Kontaktlinsen und Hörgeräte fallen anerkanntermaßen unter diese Öffnungsklausel. Im dermatologischen Bereich ist denkbar, einzelne im Zusammenhang mit der ärztlichen Therapie stehende Pflegemittel unter die Öffnungsklausel des $\S 3$ Abs. 2 Berufsordnung zu ziehen. Entscheidungen hierzu liegen bislang nicht vor.

Im Übrigen hat der Dermatologe bei seinem gewerblichen Engagement aufgrund der berufsrechtlichen Vorgaben, aber auch wegen des Steuerrechts auf eine ausreichende räumliche, organisatorische und personelle Trennung des Institutes von seiner Praxis zu achten. Von den Ärztekammern wird nach wie vor das Postulat aufgestellt, dass das Kosmetikinstitut räumlich von der Praxis zu trennen ist. Idealerweise besteht ein eigener Eingang. Ob ein gemeinsamer Empfangsbereich zulässig ist, ist nach wie vor umstritten.

\section{Ende des ausgelagerten Praxisraums}

Die Novellierung der Musterberufsordnung für Ärzte aus dem Jahr 2004 hebt die bislang strenge Bindung der ärztlichen Tätigkeit an die Niederlassung in eigener Praxis und die ehemaligen komplizierten Regelungen zur Zweigpraxis und ausgelagerten Praxisräumen auf. Nach ihrer Umsetzung in Landesberufsordnungen wird es dem Arzt gestattet sein, neben seiner Praxis an in bis zu 2 weiteren Orten ärztlich tätig zu werden. Die Integration eines ausgelagerten Praxisraumes zur Erbringung ärztlicher Leistungen in ein Kosmetikinstitut wird damit erleichtert werden.

\section{Risiko Gewerbesteuer}

Auch bezüglich der Steuern werden an die kosmetische Tätigkeit des Dermatologen in Praxis und Institut neue Anforderungen gestellt. Zu beachten ist zum einen die so genannte „Abfärbetheorie“. Danach kann das Angebot gewerblicher Leistungen durch eine ärztliche Gemeinschaftspraxis zur Infektion der freiberuflichen Einkünfte mit der Gewerbesteuerpflicht führen. Um dies zu vermeiden, ist streng darauf $\mathrm{zu}$ achten, dass gewerbliche Leistungen nicht von der Gemeinschaftspraxis erbracht werden. Gegebenenfalls ist für die gewerblichen Leistungen eine neue Personengesellschaft zu gründen, die sich als Außenauftritt erkennbar von der Gemeinschaftspraxis absetzt und insbesondere über eigenes Brief- und Rechnungspapier, eigene Geschäftskonten etc. verfügt. Der in Einzelpraxis niedergelassene Dermatologe hat es hier einfacher: Das Infektionsrisiko besteht ausschließlich bei Personengesellschaften.

\section{Kosmetische Dermatologie und Umsatzsteuerpflicht}

Seit 2003 ist ferner die Umsatzsteuerpflicht für kosmetisch indizierte dermatologische Leistungen zu beachten. Während die Finanzverwaltung die Umsatzsteuerbefreiung für Heilbehandlungen nach $\S 14$ Ziff. 14 UStG dahingehend interpretierte, dass sämtliche Leistungen des Arztes in Ausübung seiner ärztlichen Tätigkeit in der Praxis unabhängig von den medizinischen Indikationen von der Umsatzsteuer befreit sind, wird die Umsatzsteuerbefreiung heute aufgrund einer Entscheidung des Europäischen Gerichtshofes vom 14. 9. 2000 dahingehend beschränkt, dass lediglich solche Leistungen von der Umsatzsteuer befreit sind, die der medizinischen Betreuung von Personen durch das Diagnostizieren und Behandeln von Krankheiten dienen. Von der deutschen Finanzverwaltung werden diese Grundsätze seit dem 7. 4. 2003 angewandt mit der Folge, dass ärztliche Leistungen, die aufgrund kosmetischer Indikationen erbracht werden, umsatzsteuerpflichtig sind. In der entsprechenden Honorarberechnung, die für alle ärztlichen Leistungen weiterhin nach der Gebührenordnung für Ärzte zu erstellen ist, ist daher zusätzlich Umsatzsteuer auszuweisen. §19 UStG kodifiziert eine Befreiung für so genannte Kleinunternehmer, die im vorangegangenen Kalenderjahr die Umsatzgrenze von Euro 17500 und im laufenden der Kalenderjahr voraussichtlich eine Umsatzgrenze von Euro 50000 nicht überschreiten. Entscheidend sind hierbei ausschließlich die Umsätze aus der nicht medizinisch indizierten Tätigkeit. Eine Zusammenrechnung mit den Umsätzen aus Heilbehandlungen findet nicht statt.

Die Zukunft wird voraussichtlich weitere Liberalisierungen des ärztlichen Berufsrechts in Hinblich auf eine stärker betriebswirtschaftlich organisierte Praxisstruktur und Kooperationen mit Leistungsanbietern aus den unterschiedlichsten Bereichen bieten. Aber auch die bestehenden Reglungen bieten ausreichenden Spielraum für ein erfolgreiches Engagement in den Schnittstellen zwischen Dermatologie und Kosmetik. Diese gilt es zu nutzen! 